\title{
Fuzzy Cognitive Maps with Rough Concepts
}

\author{
Maikel León*', Benoît Depaire, and Koen Vanhoof \\ Hasselt University, Diepenbeek, Belgium \\ maikelleon@gmail.com
}

\begin{abstract}
Artificial Intelligence has always followed the idea of using computers for the task of modelling human behaviour, with the aim of assisting decision making processes. Scientists and researchers have developed knowledge representations to formalize and organize such human behaviour and knowledge management, allowing for easy translation from the real world, so that the computers can work as if they were "humans". Some techniques that are common used for modelling real problems are Rough Sets, Fuzzy Logic and Artificial Neural Networks. In this paper we propose a new approach for knowledge representation founded basically on Rough Artificial Neural Networks and Fuzzy Cognitive Maps, improving flexibility in modelling problems where data is characterized by a high degree of vagueness. A case study about modelling Travel Behaviour is analysed and results are assessed.
\end{abstract}

Keywords: Rough artificial neural networks, fuzzy cognitive maps, knowledge representation, modelling problems.

\section{Introduction}

The concept of upper and lower bound has been used in a variety of applications in Artificial Intelligence. In particular, theory of rough sets has demonstrated the usefulness of upper and lower bounds in fields such as rule generation. Additional advances in rough set theory have shown that the concept of upper and lower bounds offer a wider framework that can be suitable for diverse types of applications [1].

On the other hand a Fuzzy Cognitive Map (FCM) is a combination of some aspects from Fuzzy Logic, Neural Networks and other techniques; combining the heuristic and common sense rules of Fuzzy Logic with the learning heuristics of the Neural Networks. They were introduced by Kosko [2], who enhanced cognitive maps with fuzzy reasoning, that had been previously used in the field of socio-economic and political sciences to analyse social decision-making problems.

The use of FCM for many applications in different scientific fields was proposed, they had been apply to analyse extended graph theoretic behaviour, to make decision analysis and cooperate distributed agents, also were used as structures for automating human problem solving skills and as behavioural models of virtual worlds, etc.

This paper proposes rough patterns for simulations using FCM. Each value in a rough pattern is a pair of upper and lower bound. Conventional FCM models

\footnotetext{
* Corresponding author.
} 
generally use a precise input pattern in their estimations. The conventional FCM models need to be modified to accommodate rough patterns. Rough concepts proposed in this paper provide an ability to use rough patterns. Each rough concept stores the upper and lower bounds of the input and output values.

Depending upon the nature of the application, two rough concepts in the net can be connected to each other using either two approaches, using the idea of Rough Artificial Neural Networks (RANN), which have been studied in literature in many aspects. Then, a Fuzzy Cognitive Map with Rough Concepts (RFCM) consists of a combination of rough and conventional concepts connected each other, considering the hybridization as an approach together with other appropriately defined methods, e.g., fuzzy logic and domain-specific analytical techniques.

Hybrid technique such as rough-fuzzy had been attracting great attentions of many researchers since a while, and several examples have shown that the hybrid techniques perform better than the non-hybrid in a huge amount of scientific fields.

\section{Rough Artificial Neural Networks}

Generalizations of neurons have been followed by generalizations of the entire network structures and corresponding learning mechanisms. New models of neural networks have been studied more and more often as hierarchical structures of complex concepts (granules), which finally resulted in the methodology of roughneural computing:

- Construction of systems performing complex tasks using simple rough neurons and their straightforward generalizations transforming parameters of concepts.

- Hierarchical structure that represents gradual formation of more complex granules (concepts) modelling complex phenomena or structures, or projection onto simpler granules (concepts) modelling aggregation of information, conflict resolution etc.

- Flexibility and robustness originating in highly adjustable structure of possibly generalized rough neurons, their connections, and intermediate transformations enabling to vary the structures of granules (concepts) throughout the network.

- Ability to learn from examples a desired setting of the network weights, just like in case of standard neural network models, in particular ability to adapt the mechanism of backpropagation for networks involving complex granules and neurons.

Rough set theory introduced by Pawlak in 1982 is a mathematical tool to deal with vagueness and uncertainty of information [3]. The theory of rough sets has demonstrated the usefulness of upper and lower bound, so, the concept of upper and lower bound has been used in a variety of applications in intelligent system. Driven by the idea of decomposing the set of all objects into upper and lower bond, it was introduced the idea of rough neuron to construct RANN.

Each neuron $\mathrm{R}$ is a pair, for the upper bound $\mathrm{R}^{*}$ and for the lower bound $\mathrm{R} *$. Those two neurons can exchange information between each other and between other rough (conventional) neuron. Rough neurons proposed [4] provide an ability to use rough 
patterns, which are based on the notion of rough values. It is possible to use a rough neuron to successfully characterize a range or values set for variables such as age, weight or temperature.

\section{Fuzzy Cognitive Maps}

FCM in a graphical illustration seem to be a signed directed graph with feedback, consisting of nodes and weighted arcs (see figure 1).
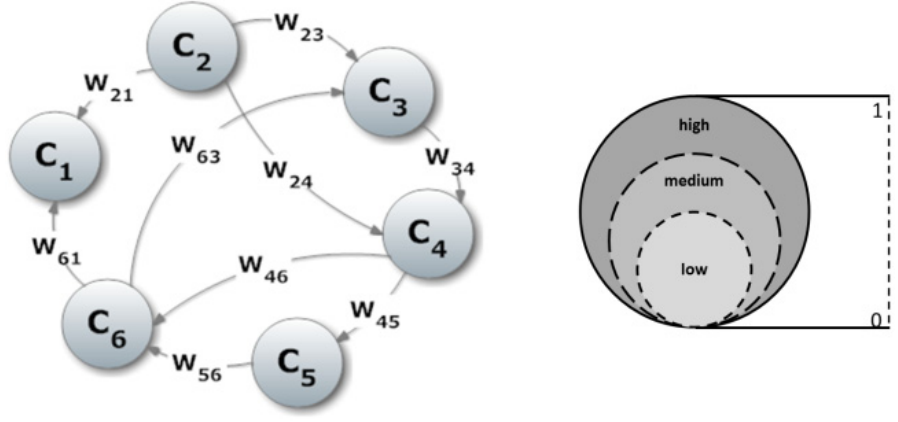

Fig. 1. Simple Fuzzy Cognitive Map. Concept activation level.

Graph nodes place for the concepts that are used to characterize the system behaviour and they are connected by signed and weighted arcs showing the causal relationships that connect the concepts. It must be mentioned that the values in the graph are fuzzy, so concepts take values in the range between $[0,1]$ and the weights of the arcs are in the interval $[-1,1]$. The weights of the $\operatorname{arcs}$ between concept $C_{i}$ and concept $\mathrm{C}_{\mathrm{j}}$ could be positive $\left(\mathrm{W}_{\mathrm{ij}}>0\right)$ which means that an augment in the value of concept $C_{i}$ leads to the increase of the value of concept $C_{j}$, and a decrease in the value of concept $\mathrm{C}_{\mathrm{i}}$ leads to a reduce of the value of concept $\mathrm{C}_{\mathrm{j}}$. Or there is negative causality $\left(\mathrm{W}_{\mathrm{ij}}<0\right)$ which means that an increase in the value of concept $\mathrm{C}_{\mathrm{i}}$ leads to the decrease of the value of concept $\mathrm{C}_{\mathrm{j}}$ and vice versa [5].

Observing this graphical representation, it becomes clear which concept influences other concepts showing the interconnections between concepts and it permits updating in the construction of the graph. Each concept represents a characteristic of the system; in general it stands for events, actions, goals, values, trends of the system that is modelled, etc. Each concept is characterized by a number that represents its value and it results from the renovation of the real value of the system's variable.

Beyond the graphical representation of the FCM there is its mathematical model. It consists of a $1 \times n$ state vector $\mathrm{A}$ which includes the values of the $\mathrm{n}$ concepts and a $\mathrm{n} \times \mathrm{n}$ weight matrix $\mathrm{W}$ which gathers the weights $\mathrm{W}_{\mathrm{ij}}$ of the interconnections between the $\mathrm{n}$ concepts. The value of each concept is influenced by the values of the connected concepts with the appropriate weights and by its previous value. So the value $A_{i}$ for each concept $\mathrm{C}_{\mathrm{i}}$ can be calculated, among other possibilities, by the following rule expressed in (1). 


$$
A_{i}=f\left(\sum_{\substack{j=1 \\ j \neq i}}^{n}\left[A_{j} \times W_{j i}\right]\right)
$$

Where $A_{i}$ is the activation level of concept $C_{i}, A_{j}$ is the activation level of concept $\mathrm{C}_{\mathrm{j}}$ and $\mathrm{W}_{\mathrm{ij}}$ is the weight of the interconnection between $\mathrm{C}_{\mathrm{j}}$ and $\mathrm{C}_{\mathrm{i}}$, it is to say, the value of $\mathrm{A}_{\mathrm{i}}$ depends of the weighted sum of its input concepts, and $f$ is a transfer, threshold or normalization function, used over concept value to decrease unbounded inputs to a severe range. This destroys the possibility of quantitative results, but it gives us a basis for comparing nodes (on or off, active or inactive, etc.). This mapping is a variation of the "fuzzification" process in fuzzy logic, giving us a qualitative model and frees us from strict quantification of edge weights.

So the new state vector $A_{\text {new }}$ is computed by multiplying the previous state vector $\mathrm{A}_{\text {old }}$ by the weight matrix $\mathrm{W}$, see (2). The new vector shows the effect of the change in the value of one concept in the whole FCM [6].

$$
A_{\text {new }}=f\left(A_{\text {old }} \times W\right)
$$

In order to build an FCM, the knowledge and experience of one expert on the system's operation must be used. The expert determines the concepts that best illustrate the system; a concept can be a feature of the system, a state or a variable or an input or an output of the system; identifying which factors are central for the modelling of the system and representing a concept for each one. When the experts have observed which system elements influence others; they must determine the effect among concepts, with a fuzzy value per interconnection, due to it has been reflected that there is a causation fuzzy degree between two connected concepts.

FCM feedback structure also makes a distinguishing from the earlier forward-only acyclic cognitive maps and from modern Artificial Intelligence expert-system search trees. Such tree structures are not dynamical systems because they lack edge cycles or closed inference loops. Nor are trees closed under combination. Combining several trees does not produce a new tree in general because cycles or loops tend to occur as the number of combined trees increases.

\section{Introducing Rough Concepts in Fuzzy Cognitive Maps}

When a FCM has been constructed, it can be used to model and simulate the behaviour of the system. Firstly, the FCM should be initialized, the activation level of each of the nodes of the map takes a value based on expert's opinion for the current state and then the concepts are free to interact. This interaction between concepts continues until a fixed equilibrium is reached; a limited cycle is reached or a chaotic behaviour is exhibited. So, FCM are a powerful methodology that can be used for modelling systems, avoiding many of the knowledge extraction problems which are usually present in by rule based systems. 
It is possible to have better results in the drawing of the FCM, if more than one expert is used. In that case, all experts are polled together and they determine the relevant factors and thus the concepts that should be presented in the map. Then, experts are individually asked to express the relationship among concepts; during the assigning of weights, three parameters must be considered: how strongly concepts influence each other, what is the sign of the weight and whether concepts cause.

But is not always easy the initialization process, and how to fix a value or how to make different experts agree? In a rough pattern, the value of each variable is specified using lower and upper bounds, using the idea from RANN [7]. But also, there is a consequence, related to the links, so figure 2 shows how to solve this problem. If the rough concept A excites the activity of B (i.e. increase in the output of $A$ will result in the increase in the output of $B$ ), then $A^{*}$ will be connected to $B^{*}$ and $\mathrm{A}^{*}$ will be connected to $\mathrm{B}^{*}$.

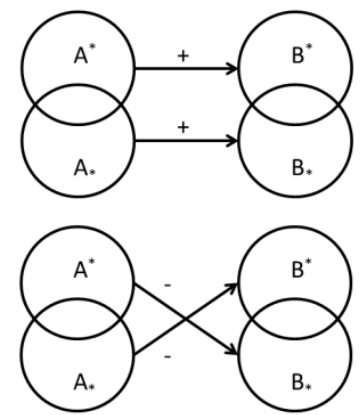

Fig. 2. Connections between rough concepts

On the other hand, if $\mathrm{A}$ inhibits the activity of $\mathrm{B}$ (i.e. increase in the output of $\mathrm{A}$ corresponds to the decrease in the output of $\mathrm{B}$ ), then $\mathrm{A}^{*}$ will be connected to $\mathrm{B}_{*}$ and $A *$ will be connected to $B^{*}$ [8]. So now formula (1) cannot be applied, so a modification for the inference process is needed. Formulas (3), (4) and (5) will describe the necessary readjustment.

$$
\begin{gathered}
\operatorname{input}\left(\mathrm{A}_{\mathrm{i}}\right)=\sum_{\substack{\mathrm{j}=1 \\
\mathrm{j} \neq \mathrm{i}}}^{\mathrm{n}}\left[\operatorname{output}\left(\mathrm{A}_{\mathrm{j}}\right) \times \mathrm{W}_{\mathrm{ji}}\right] \\
\operatorname{output}\left(A^{*}\right)=\max \left(f\left(\operatorname{input}\left(A^{*}\right)\right), f\left(\operatorname{input}\left(A_{*}\right)\right)\right) \\
\operatorname{output}\left(A_{*}\right)=\min \left(f\left(\operatorname{input}\left(A^{*}\right)\right), f\left(\operatorname{input}\left(A_{*}\right)\right)\right)
\end{gathered}
$$

There are different possibilities to choose a transfer function, one of the most used in literature appears in (6), but many others can be also used.

$$
f(x)=\frac{1}{1+e^{-9(x-0.5)}}
$$


To emphasize it is illustrated figure 3, showing the transformation from a classical FCM into a RFCM.
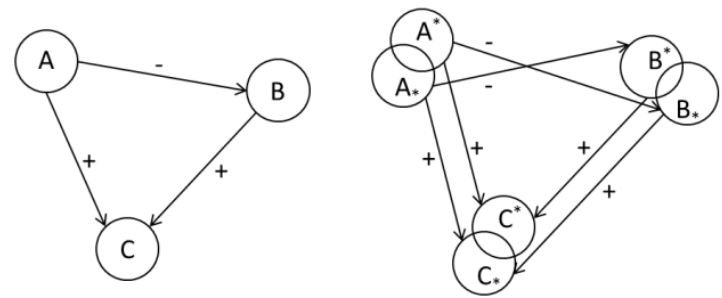

Fig. 3. FCM and the corresponding RFCM

But if the output in general of the rough concept A is desired, it can be computed using formula (7).

$$
A=\frac{A^{*}-A_{*}}{\operatorname{average}\left(A^{*}, A_{*}\right)}
$$

\section{$5 \quad$ Workbench for Modelling Complex Systems Based on FCM and RFCM}

The scientific literature shows some software products developed with the intention of drawing FCM by non-expert in computer science, as FCM Modeler [9] and FCM Designer [10]. The first one is a rustic incursion, while the second one is a better implementation, but still hard to interact with and almost without experimental facilities. Figure 4 shows the general architecture of our proposing workbench to model and simulate FCM, having the facility of using also Rough Concepts, in real applications where is extremely difficult to set up an initial point of the system, and a upper and lower bounds are easily defined.

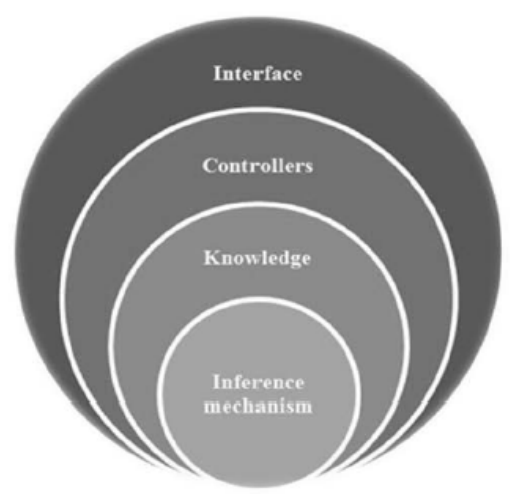

Allows the user-tool interaction through the options to create FCM, definition of parameters and formalization of the information into a knowledge base.

Makes a link between the Interface and the algorithms and data, it is a connectivity layer that guarantees a right manipulation of the information.

Generates the computational representation of the created FCM from an Artificial Intelligence point of view. Processes the input and output data of algorithms in the variables modeling.

Makes the inference process through the mathematical calculus for the prediction of the variable values.

Fig. 4. General architecture of the proposed workbench 
In figure 5 it is possible to observe the main window of the workbench, in the interface appears some facilities to create concepts, make relations, and define parameters, also to initialize the execution of the inference process, and visualization options for a better understanding of the simulation process.

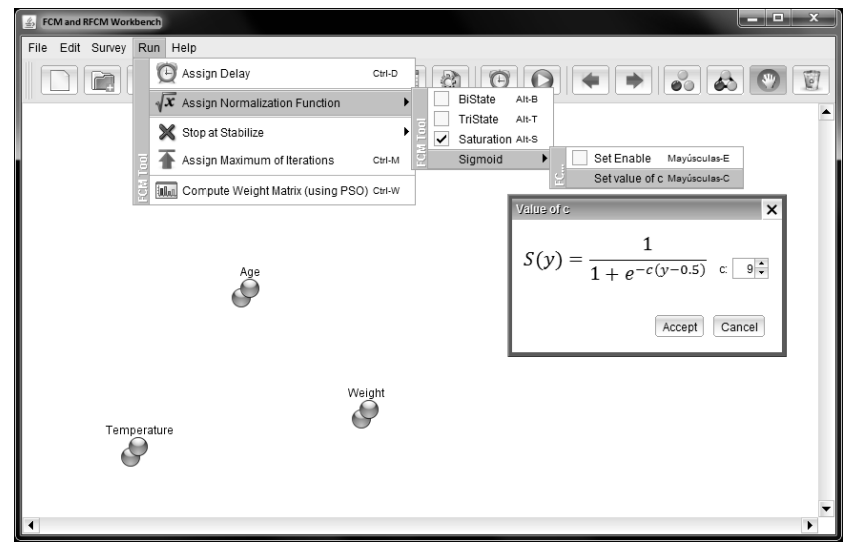

Fig. 5. Main view and Run options of the Workbench

There were defined some facilities and options in the tool, to create, open or save an FCM or RFCM. Through these amenities a non-expert in Computer Science is able to elaborate FCM or RFCM describing systems; we had paid attention to these facilities in order to guaranty that the workbench is usable for simulation and experimentation purposes. It is exposed some important options, where is possible to define the assignment of a delay time in the execution for a better understanding of the running of the FCM or RFCM in the inference process, also it is possible to define the transfer function that the FCM will use in the running.

In simulation experiments the user can compare results using these different functions or just can select the appropriate function depending of the problem to model:

- Binary FCM are suitable for highly qualitative problems where only representation of increase or stability of a concept is required.

- Trivalent FCM are suitable for qualitative problems where representation of increase, decrease or stability of a concept is required.

- Sigmoid FCM are suitable for qualitative and quantitative problems where representation of a degree of increase, a degree of decrease or stability of a concept is required and strategic planning scenarios are going to be introduced.

\section{Case Study: Modelling Travel Behaviour}

Transport Demand Management (TDM) is of vital importance for decreasing travelrelated energy consumption and depressing high weight on urban infrastructure. TDM, or also known as "mobility management", is a term for measures or strategies 
to make improved use of transportation means by reducing travel demand or distributing it in time and space. Many attempts have been made to enforce TDM that would influence individuals unsustainable travel behaviour towards more sustainable forms, TDM can be effectively and efficiently implemented if they are developed founded on a profound understanding of the basic causes of travel, such as people's reasons and inclinations and comprehensive information of individuals behaviours.

In the process of transportation planning, TDM forecast is one of the most important analysis instruments to evaluate various policy measures aiming at influencing travel supply and demand. In past decades, increasing environmental awareness and the generally accepted policy paradigm of sustainable development made transportation policy measures shift from facilitation to reduction and control.

Objectives of travel demand management measures are to alter travel behaviour without necessarily embarking on large-scale infrastructure expansion projects, to encourage better use of available transport resources avoiding the negative consequences of continued unrestrained growth in private mobility.

Individual activity travel choices can be considered as actual decision problems, causing the generation of a mental representation or cognitive map of the decision situation and alternative courses of action in the expert's mind. This cognitive map concept is often referred to in theoretical frameworks of travel demand models, especially related to the representation of spatial dimensions, but much features can be taken into account (see figure 6).

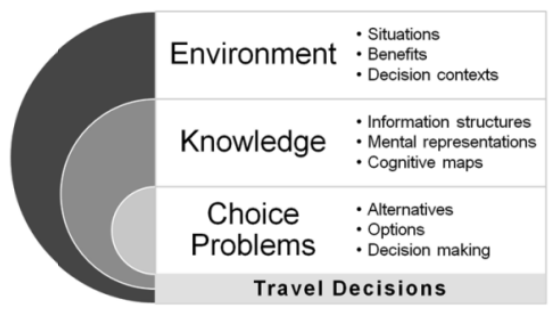

Fig. 6. Abstraction levels of mind related to Travel Behaviour

However, actual model applications are scarce, mainly due to problems in measuring the construct and putting it into the model's operation. The development of the mental map concept can benefit from the knowledge provided by individual tracking technologies. Researches are focusing on that direction, in order to improve developed models and to produce a better quality of systems. At an individual level it is important to realize that the relationship between travel decisions and the spatial characteristics of the environment is established through the individual's perception and cognition of space. As an individual observes space, for instance through travel, the information is added to the individual's mental maps.

Records regarding individual's decision making processes can be used as input to generate mental models. Such models treat each individual as an agent with mental qualities, such as viewpoints, objectives, predilections and inclinations. For the modelling of such models, several artificial intelligence techniques can be used, in 
this case FCM and RFCM will be study. They try to genuinely simulate individual's decision making processes. Consequently, they can be used not only to understand people's travel behaviours, but also to pretend the changes in their actions due to some factors in their decision atmosphere.

During a decision making process, a decision maker activates a temporary mental representation in his working memory based on his previous experiences or existing knowledge. Therefore, constructing a mental representation requires a decision maker to recall, reorder and summarize relevant information in his long-term memory. It may involve translating and representing this information into other forms, such as a scheme or diagram, supporting coherent reasoning in a connected structure.

In the city of Hasselt, capital of the Flemish province of Limburg, Belgium, a study related to Travel Behaviour was made. The city has a population around 72000 habitants, with a traffic junction of important traffic arteries from all directions. Hasselt is surrounded by 2 ring roads, the bigger one serves to retain traffic out and the small one helps to keep traffic out of the commercial centre, being almost totally a pedestrian area.

In our experiment more than 220 real habitants were asked to specify how they take into account the transport mode they will use for an imaginary shopping activity: situation, attribute and benefit variables; and starting from that data, a FCM and RFCM structures per person were developed. At the same time, virtual scenarios were presented, and the personal decisions of each individual were stored.

Both models (FCM and RFCM) were created per person, and the scenarios were played. Figure 7 presents the performances of the computational models. Although the FCM models performed $92.68 \%$, and it is considered a good result, RFCM models performed $97.1 \%$, being significantly better.

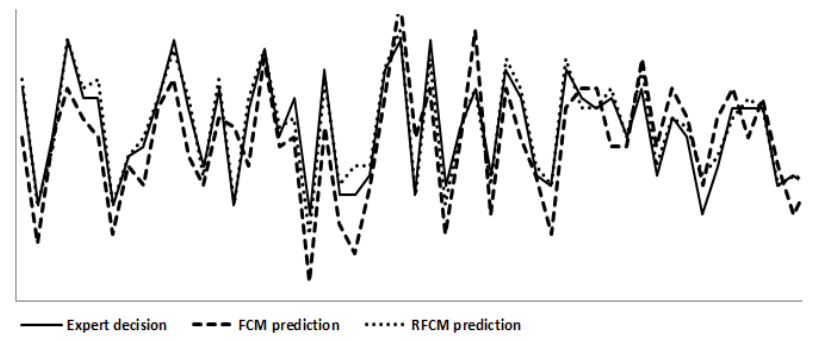

Fig. 7. FCM vs. RFCM prediction

In table 1 are indicated other attributes of the comparison, to have more detailed information of the experiment execution. Measures indicate a major discharge of the RFCM models.

Table 1. Attributes of the comparison

\begin{tabular}{c|cc} 
& FCM models & RFCM models \\
\hline Accuracy & $92.68 \%$ & $97.1 \%$ \\
Oversized estimation & $11.06 \%$ & $05.57 \%$ \\
Undersized estimation & $13.07 \%$ & $04.79 \%$ \\
Interpretable & Yes & Yes \\
Simpler structure & $(\mathrm{X})$ & $($ ) \\
Easier initialization & $(\mathrm{X})$
\end{tabular}


It is considered a substantial result, given by having structures able to simulate how people think when a transport mode decision and area election for specific activity is asked, offering policy makers a tool to play with, in order to test new policies, and to know in advance the possible resounding in the society.

\section{Conclusions}

In this paper, we presented cooperative rough pattern and FCM techniques. A rough concept can be view as a pair of concepts supporting upper and lower bounds as opposed to precise values, exchanging information with each other during the calculation of their outputs. The development of a workbench based on FCM and RFCM for the modelling of complex systems was presented, showing facilities for the creation of FCM and RFCM, and options to make the inference process comprehensible and used for simulations experiments. A better performance of RFCM over FCM was obtained in a real world modelled problem. In the presented case study a social and politic repercussion is evident, as we offer to policymakers a framework and real data to play with, in order to study and simulate individuals behaviour for city infrastructure development and demographic planning.

\section{References}

1. Chandana, S., Mayorga, S.: Rough Approximation based Neuro-Fuzzy Inference System. In: IEEE HIS, pp. 518-521 (2005)

2. Kosko, B.: Fuzzy Cognitive Maps. International Journal of Man-Machine Studies 24, 65-75 (1986)

3. Pawlak, Z.: Rough classification. International Journal of Information and Computer Sciences, 145-172 (1982)

4. Lingras, P.: Rough neural networks. In: IPMU International Conference on Information Processing and Management of Uncertainty in Knowledge-based Systems, pp. 1445-1450 (1996)

5. Wei, Z.: Using fuzzy cognitive time maps for modelling and evaluating trust dynamics in the virtual enterprises. In: Expert Systems with Applications, pp. 1583-1592. Elsevier Ltd. (2008)

6. León, M., Nápoles, G., Rodriguez, C., García, M.M., Bello, R., Vanhoof, K.: A Fuzzy Cognitive Maps Modeling, Learning and Simulation Framework for Studying Complex System. In: Ferrández, J.M., Álvarez Sánchez, J.R., de la Paz, F., Toledo, F.J. (eds.) IWINAC 2011, Part II. LNCS, vol. 6687, pp. 243-256. Springer, Heidelberg (2011)

7. Ming, H., Boqin, F.: Extracting Classification Rules with support rough neural networks. In: Torra, V., Narukawa, Y., Miyamoto, S. (eds.) MDAI 2005. LNCS (LNAI), vol. 3558, pp. 194-202. Springer, Heidelberg (2005)

8. Pal, L., Polkowski, S.: Rough-Neural Computing. Cognitive Technologies Series. Springer (2004)

9. Mohr, S.: Software Design for a Fuzzy Cognitive Map Modelling Tool. Tensselaer Polytechnic Institute (1997)

10. Contreras, J.: Aplicación de MCDD a tareas de supervisión y control. Universidad de los Andes. Mérida, Venezuela (2005) 